\title{
Rheumatoid arthritis, anti-tumour necrosis factor therapy, and risk of malignant melanoma: nationwide population based prospective cohort study from Sweden
}

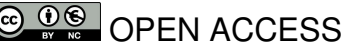

\author{
Pauline Raaschou consultant in clinical pharmacology ${ }^{12}$, Julia F Simard assistant professor ${ }^{1}$, Marie \\ Holmqvist resident ${ }^{1}$, Johan Askling professor and consultant in rheumatology ${ }^{13}$, for the ARTIS Study \\ Group
}

${ }^{1}$ Clinical Epidemiology Unit, Department of Medicine Solna, Karolinska Institutet, SE-171 76, Stockholm, Sweden; ${ }^{2}$ Clinical Pharmacology Unit, Department of Medicine Solna, Karolinska Institutet; ${ }^{3}$ Rheumatology Unit, Department of Medicine Solna, Karolinska Institutet

\begin{abstract}
Objectives To investigate the potential association between tumour necrosis factor (TNF) inhibitor treatment and malignant melanomas in rheumatoid arthritis, melanoma risks in rheumatoid arthritis patients not treated with biological drugs, and risk of all site cancer with TNF inhibitors as used in rheumatoid arthritis.

Design Population based cohort study.

Setting Prospectively recorded data from national clinical, health, and demographic registers in Sweden 2001-10.

Patients with rheumatoid arthritis treated $(n=10$ 878) or not $(n=42$ 198) with TNF inhibitors and matched general population comparators $(n=162$ 743).

Main outcome measures The primary outcome was first invasive melanoma in people without any history of invasive cancer of any type. Hazard ratios were estimated using Cox regression, comparing non-biological drug treated rheumatoid arthritis patients with the general population comparator and TNF inhibitor treated rheumatoid arthritis patients with those not treated with biological drugs. Secondary outcomes included in situ melanomas, second primary melanomas, and all site cancer.

Results 113 first invasive melanomas occurred in rheumatoid arthritis patients not treated with biological drugs, and 393 occurred in the general population comparator cohort. Rheumatoid arthritis patients not treated with biological drugs were not at significantly increased risk of melanoma compared with the general population (hazard ratio 1.2, 95\% confidence interval 0.9 to 1.5$)$. 38 first invasive melanomas occurred in rheumatoid arthritis patients treated with TNF inhibitors; these patients had an increased risk of melanoma compared with rheumatoid arthritis patients not treated with biological drugs (hazard ratio $1.5,1.0$ to $2.2 ; 20$ additional
\end{abstract}

cases per 100000 person years). The risk of a second primary melanoma was non-significantly increased (hazard ratio $3.2,0.8$ to 13.1 ; $\mathrm{n}=3 v 10$ ) in rheumatoid arthritis patients treated with TNF inhibitors compared with those not treated with biological drugs.

Conclusion Overall, patients with rheumatoid arthritis who have not been treated with biological drugs are not at increased risk of invasive melanoma compared with the general population. Rheumatoid arthritis patients selected for TNF inhibitor treatment are not at increased overall risk for cancer but have a $50 \%$ increased relative risk of invasive melanoma. Given the small increase in absolute risk, these finding may not markedly shift the overall risk-benefit balance of TNF inhibitors as used in clinical practice but might do so in patients at high risk of melanoma for other reasons.

\section{Introduction}

Activation of the immune system is a key event in the tumour defence triggered by malignant melanoma (referred to hereafter as melanoma). ${ }^{1}$ States of impaired immune competence, such as in AIDS and organ transplantation, have been identified as risk factors for melanoma. ${ }^{2-4}$ Rheumatoid arthritis, non-biological drug treatments, or both, might suppress tumour surveillance and in theory increase the risk of melanoma. ${ }^{56}$

Introduced in the late 1990s, tumour necrosis factor (TNF) inhibitors have become a mainstay in the treatment of rheumatoid arthritis. Although the overall risk of cancer seems not to be increased, ${ }^{7-12}$ TNF inhibitor treatment has been feared to increase the risk of melanoma. ${ }^{5}$ Experimental studies indicate that TNF plays an important role in the growth regulation of melanomas, ${ }^{13}$ and recent clinical data link relapse of melanoma 
with low concentrations of TNF. ${ }^{14}$ Isolated limb perfusion with TNF is a therapeutic approach used in advanced melanoma, supporting the notion of TNF as a protective cytokine in certain clinical manifestations of melanoma. ${ }^{15} 16$

As supported by a recent review, most, ${ }^{17-19}$ but not all, ${ }^{6} 11$ observational studies report no increased risk of melanoma in patients with rheumatoid arthritis not treated with biological drugs compared with the general population (supplementary table A).$^{20}$ In rheumatoid arthritis patients treated with TNF inhibitors, case reports and at least one observational study suggest a possible link between TNF inhibitors and risk of melanoma. ${ }^{1021-23}$ Furthermore, a conspicuous number of second or recurrent melanomas have been reported in TNF inhibitor treated rheumatoid arthritis patients with a history of melanoma before starting treatment. ${ }^{24}$ In an interim analysis based on the Swedish biologics register, we previously reported, in abstract format and based on a small number of events, an excess risk of melanoma in rheumatoid arthritis patients treated with TNF inhibitors. ${ }^{25}$

This study represents a considerable extension of our interim analysis. Using a nationwide population based cohort design, we investigated risks of melanoma in patients with rheumatoid arthritis compared with the general population and whether patients selected for treatment with TNF inhibitors were at particularly increased risk.

\section{Methods}

\section{Study design}

This was a population based cohort study of rheumatoid arthritis patients and matched general population comparators. Our main exposures of interest were rheumatoid arthritis and TNF inhibitors. Our outcome of interest was melanoma.

\section{Setting}

Provision of healthcare in Sweden is independent of patient related financial capacity or insurance status. Rheumatoid arthritis patients are typically treated in public care by rheumatologists. Personal identification numbers permit linkage of information from national and virtually complete registers on demographics, morbidity, and mortality, as described elsewhere. ${ }^{26}$ Typically, and in this study, only $0.2 \%$ of all people have had to be excluded owing to inconsistent or missing data precluding unambiguous follow-up.

\section{Data sources, participants, and variables}

The Swedish outpatient register was started in 2001 as a new component of the Swedish patient register (which also covers virtually all hospital discharges since 1987). The outpatient register includes information on diagnoses in non-primary outpatient care, coded according to ICD-10 (international classification of diseases, 10th revision). ${ }^{27}$ The coverage varies with calendar year and specialty but is estimated to be nearly $90 \%$ of all rheumatoid arthritis patients in Sweden. ${ }^{28}$ Chart reviews indicate that $90 \%$ of the diagnoses of rheumatoid arthritis are correct according to the American College of Rheumatology criteria. ${ }^{29} 30$

The Swedish biologics register (Anti-Rheumatic Therapy in Sweden, ARTIS) is a subset of the Swedish rheumatology quality register. It includes adult patients with rheumatic diseases starting any anti-rheumatic biological drug treatment. At start of treatment and at follow-up visits, the treating rheumatologist enters details of the disease activity and anti-rheumatic treatment. The coverage of ARTIS is approximately $90 \% .^{31}$
The national prescribed drug register contains information on all prescribed drugs dispensed at Swedish pharmacies from July 2005 onwards, with an estimated coverage of close to $100 \% .^{32}$ The Swedish national cancer register was established in 1958. Reporting of incident cancers is mandatory, resulting in an estimated coverage of greater than $95 \% .^{33}$ The register contains data on date of cancer, type of cancer according to the ICD classification, ${ }^{27}$ and morphology. The Swedish population register includes data on residency and dates of immigration and emigration for all people ever resident in Sweden from 1961 onwards, and coverage is virtually complete. ${ }^{34}$ The Swedish cause of death register is updated annually and provides information on dates and causes of death for all deceased residents from 1952 onwards. ${ }^{35}$

\section{Study population}

Through linkage of ARTIS, the outpatient register, and the cancer register, we identified a nationwide rheumatoid arthritis cohort. We identified all patients who had never been treated with biological drugs and had a minimum of two visits listing rheumatoid arthritis as the main diagnosis or a contributory diagnosis in non-primary outpatient care between 1 January 2001 and 31 December $2010(n=49$ 136; figure $\Downarrow)$. At least one of these visits was at a department of rheumatology or internal medicine. The second visit served as the date of inclusion. We excluded patients who had ever received a diagnosis of juvenile idiopathic arthritis, ankylosing spondylitis, systemic lupus erythematosus, or psoriatic arthritis (see appendix 1 for ICD codes). We used two definitions of this non-biological drug treated rheumatoid arthritis study population. The first definition included patients with no history of any invasive cancer before inclusion into the cohort $(n=42198)$, and the other included patients with a history of melanoma before inclusion $(n=295)$. Similarly, in ARTIS, we identified those rheumatoid arthritis patients who started a TNF inhibitor as their first ever biological drug treatment between 1 January 1998 and 31 December 2010 $(n=11343$; figure $\downarrow)$. Of these, $10878 \mathrm{had}$ no history of invasive cancer at start of treatment, and 54 had a history of melanoma. More than $99 \%$ of the TNF inhibitor treated patients were also in the above mentioned nationwide rheumatoid arthritis cohort. Through linkage to the Swedish population register and the cancer register, we assembled a general population comparator cohort to the non-biological drug treated and TNF inhibitor treated rheumatoid arthritis patients, matched one to five on sex, year of birth, marital status, and county of residence ( $n=204$ 054). We assigned comparators the same date of inclusion as their matched rheumatoid arthritis patient and required them to be alive and free of invasive cancer at this date $(n=162743$; figure $\downarrow$ ).

\section{Definition of exposure}

We compared three exposure categories: non-biological drug treated rheumatoid arthritis patients, rheumatoid arthritis patients starting a first ever treatment with any of the five TNF inhibitors approved in Sweden during the study period (adalimumab, certolizumab pegol, etanercept, golimumab, and infliximab), and the general population. TNF inhibitor treated patients were considered "ever exposed" and contributed time even if the TNF inhibitor treatment was stopped or a non-TNF inhibitor biological drug was started. We treated TNF inhibitor as a time dependent covariate such that patients contributed to the non-biological drug treated group until they started their first TNF inhibitor or non-TNF inhibitor biological drug and to the TNF inhibitor treated group thereafter. 


\section{Follow-up and outcome}

Follow-up began at the first date of fulfilment of the inclusion criteria into each of the exposure categories. Follow-up ended at the earliest of death, emigration, outcome, any invasive cancer other than the outcome, and 31 December 2010. Through register linkages, we identified all registered in situ or invasive cancers in the study population from inclusion to 31 December 2010. We defined the primary outcome as invasive melanoma (in people without a history of invasive cancer of any type or site). We defined three secondary outcomes: in situ melanoma (in people without a history of invasive cancer of any type or site), second invasive or in situ melanoma (in people with a history of an invasive or in situ melanoma but without a history of any other type of invasive cancer), and invasive cancer of any type and site other than basal cell skin cancer. The third secondary outcome was chosen to put the effect of any increased risk of melanoma into context. For each analysis, we excluded people not at risk and censored those no longer at risk.

\section{Chart review}

We reviewed charts to validate the diagnoses and examine the temporal order of exposure and outcome among the TNF inhibitor treated patients. We reviewed charts before updating the latest register linkage, so we examined the records for only 24 of the 39 cases of melanoma.

\section{Potential confounders}

We identified potential confounders through register linkages: country of birth, family history of melanoma, educational level, personal history of non-melanoma skin cancer in situ, hospital admissions/outpatient visits for knee/hip joint replacement surgery, chronic obstructive pulmonary disease, ischaemic heart disease, and diabetes mellitus (full description and ICD codes are in appendix 2). To adjust for exposure to non-biological disease modifying anti-rheumatic drugs, we used data from the prescribed drug register for the subset of our population followed from July 2005 through 2010 . We calculated accumulated use of methotrexate in each patient by summing of all time periods during follow-up for which a prescription was filled.

\section{Statistical analyses}

We used Cox regression to estimate hazard ratios, using follow-up time as the timescale. Alternative timescales and model specifications yielded virtually identical results.

In the analyses of TNF inhibitor treated versus non-biological drug treated rheumatoid arthritis patients, we adjusted hazard ratios for age at inclusion, sex, year of inclusion, and the potential confounders listed above. We estimated hazard ratios overall and separately by age at start of follow-up, calendar period of starting TNF inhibitor, and time since start of first TNF inhibitor. We adjusted analyses of non-biological drug treated rheumatoid arthritis patients versus the general population comparator for age and sex but not for other covariates, as these mostly pertained to time points after onset of rheumatoid arthritis.

We tested the proportional hazards assumption (and found it not to be violated) by introducing an interaction term of exposure and log of follow-up time in the model. To assess the robustness of the hazard ratios for risk of melanoma in relation to the definition of the non-biological drug treated rheumatoid arthritis comparator, we did sensitivity analyses considering three sub-cohorts nested within the original nationwide rheumatoid arthritis cohort: rheumatoid arthritis patients changing non-biological disease modifying anti-rheumatic drug treatment, rheumatoid arthritis patients stable on methotrexate, and incident rheumatoid arthritis patients starting a first ever non-biological disease modifying anti-rheumatic drug (supplementary table B).

We used Cox regression to explore predictors of risk of melanoma within the TNF inhibitor treated cohort. We assessed the following predictors at the start of treatment: age, sex, duration of rheumatoid arthritis, rheumatoid factor, and non-biological disease modifying anti-rheumatic drugs. We used the SAS software package, version 9.2, for all analyses.

\section{Results}

Table $1 \Downarrow$ describes characteristics of the TNF inhibitor treated cohort. Median follow-up was 4.8 years; 8375 (77\%) received TNF inhibitor in combination with non-biological disease modifying anti-rheumatic drugs; of these, 7119 (85\%) used methotrexate. Among the reviewed cases, all charts were identified and all diagnoses of rheumatoid arthritis and melanoma were confirmed. In all cases, the TNF inhibitor treatment antedated the diagnosis of melanoma.

\section{Non-biological drug treated rheumatoid arthritis patients versus general population}

The proportion of melanomas classified in the national cancer register as clinical stage 1 , clinical stage $2-4$, and clinical stage missing/unclassifiable were similar in the non-biological drug treated and general population cohorts $(\mathrm{P}=0.1)$. On the basis of 113 cases during 203345 person years of follow-up in the non-biological drug treated cohort (56 per 100000 ) and 393 cases during 854111 person years in the general population comparator cohort (46 per 100000 ), the age and sex adjusted hazard ratio for invasive melanoma was 1.2 (95\% confidence interval 0.9 to 1.5 ) (table $2 \Downarrow$ ).

On the basis of 57 cases of in situ melanoma during 197754 person years in the non-biological treated cohort (29 per 100 $000)$ and 219 cases during 838548 person years in the general population comparator cohort (26 per 100000 ), the age and sex adjusted hazard ratio for in situ melanoma was 1.2 (0.9 to 1.7).

On the basis of on 2788 first invasive all site cancer during 196 826 person years in the non-biological treated cohort (1416 per $100000)$ and 9736 first invasive all site cancer during 831297 person years in the general population cohort (1171 per 100 $000)$, the age and sex adjusted hazard ratio was 1.1 (1.1 to 1.2), even after exclusion of invasive melanomas from the all site analysis (data not shown).

\section{TNF inhibitor treated versus non-biological drug treated rheumatoid arthritis patients}

The proportion of melanomas classified in the national cancer register as clinical stage 1, clinical stage $2-4$, and clinical stage missing/unclassifiable were similar in the TNF inhibitor and non-biological drug treated cohorts $(\mathrm{P}=0.7)$. On the basis of 38 invasive melanomas during 57223 person years in the TNF inhibitor treated cohort (68 per 100000$)$ and 113 invasive melanomas during 203345 person years of follow-up in the non-biological treated rheumatoid arthritis cohort (56 per 100 $000)$, the age and sex adjusted hazard ratio was 1.6 (1.1 to 2.5) and the fully adjusted hazard ratio was 1.5 (1.0 to 2.2) (table $3 \Downarrow)$. We detected no significant differences in the hazard ratios of invasive melanoma between different age categories, different time intervals since start of first TNF inhibitor, different categories of accumulated time on TNF inhibitor, and different 
calendar periods of start of TNF inhibitor. Compared with non-biological drug treated rheumatoid arthritis patients, the risk of melanoma in TNF inhibitor treated men (age adjusted hazard ratio $2.7,1.6$ to 4.6$)$ was higher than that in women (1.2, 0.7 to 1.9 ) (P for difference $<0.001$ ) (table $4 \Downarrow$ ).

On the basis of 558 first invasive all site cancers during 55947 person years in the TNF inhibitor treated cohort (997 per 100 000) and 2788 first invasive all site cancers during 196826 person-years in the non-biological drug treated cohort (1416 per 100000$)$, the age and sex adjusted hazard ratio was $1.0(0.9$ to 1.1$)$ and the fully adjusted hazard ratio was 1.0 (0.9 to 1.1$)$, even after exclusion of invasive melanomas from the all site analysis (data not shown).

On the basis of 11 cases of in situ melanoma during 56080 person years in the TNF inhibitor treated cohort $(20$ per 100 000) and 57 cases of in situ melanoma during 197754 person years in the non-biological drug treated cohort (29 per 100000$)$, the hazard ratio for in situ melanoma was 1.1 (0.5 to 2.1). Owing to small numbers, we abstained from further adjustments for comorbidities and other pre-specified confounders.

Fifty four TNF inhibitor treated and 295 non-biological drug treated rheumatoid arthritis patients had a history of an invasive or in situ melanoma at the start of treatment. Among these, 3 (all in situ) versus 10 (5 in situ) patients developed a new melanoma during follow-up, corresponding to an age and sex adjusted hazard ratio of 3.2 ( 0.8 to 13.1 ). Owing to small numbers, we abstained from further adjustments for comorbidities and other pre-specified confounders.

\section{Sensitivity analyses}

Sensitivity analyses using alternative definitions of the non-biological drug treated rheumatoid arthritis comparator resulted in hazard ratios for the main outcome compared with the general population ranging from 0.3 to 1.3 and hazard ratios for TNF inhibitor treated compared with non-biological drug treated rheumatoid arthritis patients ranging from 1.5 to 3.0 (supplementary table B).

To explore methotrexate as a potential confounder, we used data from the prescribed drug register from July 2005 through 2010. When we restricted the main analysis to this time period, adjustment for methotrexate did not change the hazard ratio associated with use of a TNF inhibitor. In analyses restricted to the 18923 people among the TNF inhibitor treated and non-biological drug treated patients who entered into the study after July 2005, accumulated use of methotrexate was not associated with an increased risk of invasive melanoma (hazard ratios for melanoma ranging from 0.4 to 1.3 for one year or less through greater than three years of active methotrexate use). Similarly, in the predictor analysis, neither duration of rheumatoid arthritis nor concomitant use of non-biological disease modifying anti-rheumatic drugs at the start of the TNF inhibitor treatment emerged as predictors of melanoma (supplementary table $\mathrm{C}$ ).

\section{Discussion}

With 38 incident invasive melanomas among the TNF inhibitor treated patients and 113 among the non-biological drug treated patients, our study represents the largest investigation of risks of melanoma in patients with rheumatoid arthritis treated with TNF inhibitors to date. We found a $50 \%$ increase in relative risk of invasive melanoma in rheumatoid arthritis patients treated with TNF inhibitors compared with non-biological drug treated patients. By contrast, we found no increased risk of in situ melanomas or any overall increase in risk of all site cancer.

The significance of TNF (and thereby potentially also of TNF inhibitors) in melanoma development is supported by studies in vitro and in humans. Melanoma is commonly regarded as an immunogenic tumour. This reflects its capability of causing an immune response in which cytokines such as TNF and interferon $\alpha$ are believed to have tumour neutralising potential. ${ }^{13}{ }^{36-38}$ Profound suppression of the immune system with ciclosporin, prednisolone, and azathioprine has been shown to cause eruption of multiple melanocytic naevi. ${ }^{39}$ Thus, either through a specific effect or though "generic" effects in analogy with the observed effects of immune suppressive treatment in organ transplant patients, ${ }^{2} 4041$ TNF inhibitor treatment might increase the risk of melanoma. ${ }^{5}$

Unlike previous studies of melanoma in rheumatoid arthritis or after organ transplantation, which have studied "melanoma" as the outcome, ${ }^{468101117-1912}$ we could study invasive, in situ, and recurrent melanomas separately. We found an increased risk of invasive but not of in situ melanoma with TNF inhibitor treatment. This finding could have alternative explanations, including low power of the in situ melanoma analysis. The clinical detection of in situ and invasive melanomas might have been differential with respect to exposure status, but such detection bias is perhaps more likely to have overestimated in situ melanomas among TNF inhibitor treated patients owing to increased clinical vigilance. A substantial portion of invasive melanomas evolve from healthy skin with no primary naevus lesion. Melanoma stem cells, which interplay with fibroblasts, endothelial cells, and inflammatory cells, can directly transform into invasive melanoma, bypassing the intermediate "naevus state." ${ }^{42}$ In situ melanomas and invasive melanoma might thus have different causes, and the effect of TNF inhibitor treatment might differ accordingly.

The hazard ratios for melanoma by time of follow-up and by accumulated time on active TNF inhibitor treatment did not show any marked trend over strata, although some strata were small. Among the TNF inhibitor treated patients, male sex was associated with a significantly higher risk of melanoma compared with female sex. In the general population, the incidence of melanomas is higher among men than women. ${ }^{43}$ Our findings might indicate that any effect of TNF inhibitor treatment may be more pronounced among men than women.

Although the effect was not significant, we detected a threefold higher risk of a second primary melanoma among the TNF inhibitor treated compared with non-biological drug treated rheumatoid arthritis patients. This possibly reflects a specifically strong effect of TNF inhibitors on development of melanoma in patients already at high risk of melanoma, but data must be interpreted with caution owing to small numbers. In a study on risk of relapse and new second primary cancers from the British biologics register (BSRBR), three of $17 \mathrm{TNF}$ inhibitor treated patients with a previous melanoma had recurrences or new same site primaries, compared with none of 10 in the non-biological disease modifying anti-rheumatic drugs cohort. ${ }^{24}$

\section{Strengths and limitations of study}

Our study has several strengths and limitations. Linkage of national health and census registries ensured inclusion of virtually all rheumatoid arthritis patients seeking care in Sweden, including the vast majority of adult patients in Sweden who were new users of TNF inhibitors. We identified outcomes through linkage to the virtually complete national cancer register. Chart reviews confirmed the high diagnostic accuracy 
of the diagnoses of rheumatoid arthritis and cancer among the TNF inhibitor treated patients, as well as the temporal order of start of TNF inhibitor treatment and diagnosis of cancer. We could differentiate between first and second malignancies and between invasive and in situ melanomas, and we could also put the observed risk of melanoma in relation to that of other types of cancer. We lacked information on personal history of dysplastic or multiple naevi, which are well known risk factors for melanoma. However, as dysplastic or multiple naevi are not generally perceived to be contraindications to TNF inhibitors, channelling due to these factors is less likely.

We could adjust for several risk factors for melanoma or proxies thereof; however, as in many observational studies, confounding by indication remains a concern. We cannot exclude the possibility that the increased risk of melanoma among the TNF inhibitor treated patients was partly driven by the same factors that made the patients eligible for TNF inhibitor treatment or was associated with increased cancer surveillance during treatment. On the other hand, contraindications to TNF inhibitors and the potential for detection of cancer through pre-treatment investigations before starting a TNF inhibitor might have led to a selection of patients with an a priori lower risk of melanoma.

Therapeutic immune suppression, as in organ transplant patients, has been linked to an increased risk of melanoma. ${ }^{23}$ Although such treatment regimens are qualitatively different from and quantitatively more intense than typical non-biological treatment regimens for rheumatoid arthritis, one might hypothesise that non-biological disease modifying anti-rheumatic drugs, as used in rheumatoid arthritis, could be a confounder for risk of melanoma among TNF inhibitor treated patients. An Australian cohort study of 459 rheumatoid arthritis patients treated with methotrexate before 1986 reported a threefold increased risk of melanoma compared with the general population. ${ }^{6}$ The accumulated disease activity and the spectrum of non-biological disease modifying anti-rheumatic drug use may have been substantially different from our cohort. Alternative explanations for the discrepant finding include lack of precision and effect modification by exposure to ultraviolet light (higher in Australia than in northern Europe) or skin type. In most other cohort studies of non-biological drug treated rheumatoid arthritis patients, the typical finding has been a non-elevated risk of melanoma (supplementary table A), indicating that non-biological disease modifying anti-rheumatic drugs typically used in rheumatoid arthritis are not a strong risk factor for melanoma. This is also supported by a recent meta-analysis indicating a non-increased risk of melanoma in rheumatoid arthritis patients not treated with biological drugs compared with the general population (standardised incidence ratio 1.01, $95 \%$ confidence interval 0.93 to 1.10$).{ }^{20}$ In keeping with this, the non-biological drug treated rheumatoid arthritis cohort in our study was not at significantly elevated risk of melanoma, despite a high exposure to non-biological disease modifying anti-rheumatic drugs; nor did we observe any association with, or confounding by, use of methotrexate. Over the past decade, most patients with rheumatoid arthritis have been treated with non-biological disease modifying anti-rheumatic drugs from the onset of their disease, so disease duration is likely to be a proxy for accumulated exposure to these drugs. However, in our assessments of predictors of risk of melanoma within the TNF inhibitor treated cohort, duration of rheumatoid arthritis did not emerge as a risk factor. Nevertheless, in the absence of a full treatment history since diagnosis of rheumatoid arthritis in all of our cohorts, we cannot formally rule out confounding or effect modification by non-biological disease modifying anti-rheumatic drugs.
Starting or changing treatment may in itself increase or decrease the risk of having a cancer detected. We used three alternative definitions of the non-biological drug treated rheumatoid arthritis cohort, defined on the basis of specific states of or changes in treatment. Although statistical precision was lower, the analyses of disease modifying anti-rheumatic drugs "switchers" (a new user design) and "stable" methotrexate users showed hazard ratios similar to that of our primary analysis.

TNF inhibitors were introduced in the late 1990s. The median follow-up from start of TNF inhibitor treatment in our study was 4.8 years (maximum 10 years), which might be insufficient to detect long term effects on cancer risks.

\section{Findings in relation to other studies}

Only a few clinical studies have investigated the risk of melanoma in TNF inhibitor treated rheumatoid arthritis patients. A cohort study using US and Canadian claims data investigated cancer risks in older rheumatoid arthritis patients exposed to methotrexate, biological drugs, or both. ${ }^{11}$ The authors reported a doubled risk of melanoma among rheumatoid arthritis patients overall compared with the general population (standardised incidence ratio $2.3,1.6$ to 3.2 ), but of the 29 identified melanomas only one occurred among biological drug treated patients. A US community based cohort study, including 13 001 patients with rheumatoid arthritis, of whom approximately $50 \%$ were ever treated with biological drugs, reported an increased risk of melanoma compared with the general population (standardised incidence ratio $1.7,1.3$ to 2.2 ),${ }^{10}$ largely driven by melanomas among the TNF inhibitor treated patients, with a relative risk of 2.3 (0.9 to 5.4) comparing patients treated and not treated with biological drugs. A study from the Danish biologics register reported a trend towards increased risk of melanoma among TNF inhibitor treated $(n=3347$, six melanomas) compared with non-biological drug treated rheumatoid arthritis patients $(n=3812$, three melanomas; hazard ratio $1.54,0.37$ to 6.34$){ }^{8}$

In a recent pooled meta-analysis, estimates of risk for melanomas above one were observed for etanercept and infliximab but not for adalimumab, resulting in an overall Peto odds ratio of 1.08. Based on only four melanomas observed in three randomised controlled trials of 52-104 weeks' duration, the estimate was, however, devoid of statistical precision $(95 \%$ confidence interval 0.1 to 10.2$).{ }^{12}$ Compared with observational studies, the clinical trial setting offers a balanced distribution of risk factors between treatment groups. However, strict inclusion and exclusion criteria often limit the generalisability of trial data. In the cited meta-analysis, the incidence of melanoma was 30 per 100000 person years among the TNF inhibitor treated patients and 15 per 100000 among the non-biological drug treated patients, which is less than half the incidence rate in our study. Other meta-analyses have typically not reported specifically on risk of melanoma in association with TNF inhibitors. ${ }^{44-47}$

\section{Melanoma risk in clinical perspective}

Relative risks are clinically interpretable only in light of the underlying absolute risk. The observed 68 cases per 100000 person years in the TNF inhibitor treated cohort corresponded to a number of additional cases in the order of 20 per 100000 person years. In other words, if the observed association with TNF inhibitors reflects causality, thousands of rheumatoid arthritis patients must be treated for one year for one melanoma to be attributable to the TNF inhibitor treatment. Seven per cent of all cancers in our study were melanomas. The increased risk 
of melanoma did not translate into any increase in the overall risk of cancer, which was at unity.

Against the above, our finding does not markedly shift the overall risk-benefit balance of TNF inhibitors as used in clinical practice, in that the beneficial effects of TNF inhibitor treatment will in most cases outweigh the small increase in risk of melanoma. Our finding may, however, shift this balance in patients at high risk, such as those with a history of melanoma. Given the excellent prognosis of melanomas if detected early, increased clinical vigilance is probably advisable in such patients if treatment with TNF inhibitors is considered.

\section{Conclusions}

Our study indicates that the average risk of invasive or in situ melanoma is not increased in patients with rheumatoid arthritis not treated with biological drugs. Patients selected for treatment with TNF inhibitor are at a $50 \%$ increased risk of invasive melanomas, but not of in situ melanomas or of invasive cancers overall.

Contributors: PR, JFS, MH, and JA all contributed to the acquisition and analysis of the data and the drafting of the manuscript. JA is the guarantor.

The ARTIS Study Group comprises Eva Baecklund, Lars Coster, Helena Forsblad, Nils Feltelius, Pierre Geboreck, Lennart Jacobsson, Lars Klareskog, Staffan Lindblad, Solbritt Rantapaa-Dahlqvist, Tore Saxne, and Ronald van Vollenhoven.

Funding: This study was supported by public grants from the Swedish Cancer Society, the Swedish Research Council, Stockholm County Council, and the Swedish Foundation for Strategic Research. The ARTIS Study Group conducts scientific analyses using data from the Swedish biologics register ARTIS run by the Swedish Society for Rheumatology. For the maintenance of this register, the Swedish Society for Rheumatology has received funding, independent of the conduct of these scientific analyses, from Merck, BMS, Pfizer, Abbott Laboratories, SOBI, UCB, and Roche. These entities had no influence on the data collection, statistical analyses, manuscript preparation, or decision to submit. They were allowed to comment on the findings before submission, although all final decisions resided with the investigators. All authors have completed the Unified Competing Interest form at www. icmje.org/coi_disclosure.pdf (available on request from the corresponding author) and declare: JA has received research grants from Pfizer and Astra-Zeneca as part of a public-private research consortium (COMBINE, www.combinesweden.se) and speakers' honorariums from Merck; no other relationships or activities that could appear to have influenced the submitted work.

Ethical approval: The study was approved by the ethics committee at Karolinska Institutet.

Data sharing: No additional data available.

1 Oble DA, Loewe R, Yu P, Mihm MC Jr. Focus on TILs: prognostic significance of tumor infiltrating lymphocytes in human melanoma. Cancer Immun 2009;9:3.

2 Vajdic CM, McDonald SP, McCredie MR, van Leeuwen MT, Stewart JH, Law M, et al. Cancer incidence before and after kidney transplantation. JAMA 2006;296:2823-31.

3 Moloney FJ, Comber H, O'Lorcain P, O'Kelly P, Conlon PJ, Murphy GM. A population-based study of skin cancer incidence and prevalence in renal transplant recipients. Br J Dermatol 2006;154:498-504.

4 Grulich AE, van Leeuwen MT, Falster MO, Vajdic CM. Incidence of cancers in people with HIV/AIDS compared with immunosuppressed transplant recipients: a meta-analysis. Lancet 2007;370:59-67.

5 Chakravarty EF, Farmer ER. Risk of skin cancer in the drug treatment of rheumatoid arthritis. Expert Opin Drug Saf 2008;7:539-46.

6 Buchbinder R, Barber M, Heuzenroeder L, Wluka AE, Giles G, Hall S, et al. Incidence of melanoma and other malignancies among rheumatoid arthritis patients treated with methotrexate. Arthritis Rheum 2008;59:794-9.

7 Haynes K, Beukelman T, Curtis JR, Newcomb C, Herrinton LJ, Graham DJ, et al. Tumor necrosis factor alpha inhibitor therapy and cancer risk in chronic immune-mediated diseases. Arthritis Rheum 2013;65:48-58.

8 Dreyer L, Mellemkjaer L, Andersen AR, Bennett P, Poulsen UE, Juulsgaard Ellingsen T, et al. Incidences of overall and site specific cancers in TNFalpha inhibitor treated patients with rheumatoid arthritis and other arthritides: a follow-up study from the DANBIO registry. Ann Rheum Dis 2013;72:79-82.

9 Askling J, van Vollenhoven RF, Granath F, Raaschou P, Fored CM, Baecklund E, et al. Cancer risk in patients with rheumatoid arthritis treated with anti-tumor necrosis factor alpha therapies: does the risk change with the time since start of treatment? Arthritis Rheum 2009;60:3180-9.

10 Wolfe $\mathrm{F}$, Michaud K. Biologic treatment of rheumatoid arthritis and the risk of malignancy: analyses from a large US observational study. Arthritis Rheum 2007:56:2886-95.

11 Setoguchi S, Solomon DH, Weinblatt ME, Katz JN, Avorn J, Glynn RJ, et al. Tumor necrosis factor alpha antagonist use and cancer in patients with rheumatoid arthritis. Arthritis Rheum 2006;54:2757-64.

12 Lopez-Olivo MA, Tayar JH, Martinez-Lopez JA, Pollono EN, Cueto JP, Gonzales-Crespo $\mathrm{MR}$, et al. Risk of malignancies in patients with rheumatoid arthritis treated with biologic therapy: a meta-analysis. JAMA 2012;308:898-908.

13 Lazar-Molnar E, Hegyesi $\mathrm{H}$, Toth S, Falus A. Autocrine and paracrine regulation by cytokines and growth factors in melanoma. Cytokine 2000;12:547-54.

14 Hofmann MA, Kiecker F, Kuchler I, Kors C, Trefzer U. Serum TNF-alpha, B2M and sIL-2R levels are biological correlates of outcome in adjuvant IFN-alpha2b treatment of patients with melanoma. J Cancer Res Clin Oncol 2011;137:455-62.

15 Grunhagen DJ, de Wilt JH, van Geel AN, Verhoef C, Eggermont AM. Isolated limb perfusion with TNF-alpha and melphalan in locally advanced soft tissue sarcomas of the extremities. Recent Results Cancer Res 2009;179:257-70.

16 Deroose JP, Eggermont AM, van Geel AN, Verhoef C. Isolated limb perfusion for melanoma in-transit metastases: developments in recent years and the role of tumor necrosis factor alpha. Curr Opin Oncol 2011;23:183-8.

17 Mellemkjaer L, Linet MS, Gridley G, Frisch M, Moller H, Olsen JH. Rheumatoid arthritis and cancer risk. Eur J Cancer 1996;32A:1753-7.

18 Gridley G, McLaughlin JK, Ekbom A, Klareskog L, Adami HO, Hacker DG, et al. Incidence of cancer among patients with rheumatoid arthritis. J Natl Cancer Inst 1993;85:307-11.

19 Thomas E, Brewster DH, Black RJ, Macfarlane GJ. Risk of malignancy among patients with rheumatic conditions. Int J Cancer 2000;88:497-502.

20 Perkins S, Cohen M, Rahme E, Bernatsky S. Melanoma and rheumatoid arthritis (brief report). Clin Rheumatol 2012;31:1001-3.

21 Katoulis AC, Kanelleas A, Zambacos G, Panayiotides I, Stavrianeas NG. Development of two primary malignant melanomas after treatment with adalimumab: a case report and review of the possible link between biological therapy with TNF-alpha antagonists and melanocytic proliferation. Dermatology 2010;221:9-12.

22 Manganoni AM, Zane C, Pavoni L, Farisoglio C, Sereni E, Calzavara-Pinton P. Cutaneous melanoma in patients in treatment with biological therapy: review of the literature and case report. Dermatol Online J 2011;17(8):12.

23 Fulchiero GJ Jr, Salvaggio H, Drabick JJ, Staveley-O'Carroll K, Billingsley EM, Marks JG, et al. Eruptive latent metastatic melanomas after initiation of antitumor necrosis factor therapies. J Am Acad Dermatol 2007;56(5 suppl):S65-7.

24 Dixon WG, Watson KD, Lunt M, Mercer LK, Hyrich KL, Symmons DP. Influence of anti-tumor necrosis factor therapy on cancer incidence in patients with rheumatoid arthritis who have had a prior malignancy: results from the British Society for Rheumatology biologics register. Arthritis Care Res (Hoboken) 2010;62:755-63

25 Askling J. Anti-TNF and risk of skin cancer-data from the Swedish ARTIS-registry 1998-2006 [abstract no FRI0201]. Annual European Congress of Rheumatology (EULAR), Copenhagen, 10-13 June 2009.

26 Askling J, Fored CM, Geborek P, Jacobsson LT, van Vollenhoven R, Feltelius N, et al. Swedish registers to examine drug safety and clinical issues in RA. Ann Rheum Dis 2006;65:707-12.

27 World Health Organization. International classification of disease. WHO, 1955.

28 Simard JF, Askling J, for the ARTIS Study Ggroup. Nationwide prevalence of rheumatoid arthritis and penetration of disease-modifying drugs in Sweden. Ann Rheum Dis 2011;70:624-9.

29 Baecklund E, lliadou A, Askling J, Ekbom A, Backlin C, Granath F, et al. Association of chronic inflammation, not its treatment, with increased lymphoma risk in rheumatoid arthritis. Arthritis Rheum 2006;54:692-701.

30 Aletaha D, Neogi T, Silman AJ, Funovits J, Felson DT, Bingham CO 3rd, et al. 2010 rheumatoid arthritis classification criteria: an American College of Rheumatology/European League Against Rheumatism collaborative initiative. Ann Rheum Dis 2010;69:1580-8.

31 Neovius M, Simard J, Sundstrom A, Jacobsson L, Geborek P, Saxne T, et al. Generalisability of clinical registers used for drug safety and comparative effectiveness research: coverage of the Swedish biologics register. Ann Rheum Dis 2011;70:516-9.

32 Wettermark B, Hammar N, Fored CM, Leimanis A, Otterblad Olausson P, Bergman U, et al. The new Swedish Prescribed Drug Register-opportunities for pharmacoepidemiological research and experience from the first six months. Pharmacoepidemiol Drug Saf 2007:16:726-35.

33 Barlow L, Westergren K, Holmberg L, Talbäck M. The completeness of the Swedish Cancer Register: a sample survey for year 1998. Acta Oncol 2009;48:27-33.

34 StatisticsSweden. Population. www.scb.se/Pages/SubjectArea_2442.aspx.

35 Askling J, Fored CM, Geborek P, Jacobsson LT, van Vollenhoven R, Feltelius N, et al. Swedish registers to examine drug safety and clinical issues in RA. Ann Rheum Dis 2006;65:707-12.

36 Lizee G, Radvanyi LG, Overwijk WW, Hwu P. Immunosuppression in melanoma immunotherapy: potential opportunities for intervention. Clin Cancer Res 2006;12:2359-65s.

37 Mukherji B, Chakraborty NG. Immunobiology and immunotherapy of melanoma. Curr Opin Oncol 1995;7:175-84

38 Jack A, Boyes C, Aydin N, Alam K, Wallack M. The treatment of melanoma with an emphasis on immunotherapeutic strategies. Surg Oncol 2006;15:13-24.

39 Alaibac M, Piaserico S, Rossi CR, Foletto M, Zacchello G, Carli P, et al. Eruptive melanocytic nevi in patients with renal allografts: report of 10 cases with dermoscopic findings. J Am Acad Dermatol 2003;49:1020-2.

40 Collett D, Mumford L, Banner NR, Neuberger J, Watson C. Comparison of the incidence of malignancy in recipients of different types of organ: a UK registry audit. Am J Transplant 2010;10:1889-96.

41 Kasiske BL, Snyder JJ, Gilbertson DT, Wang C. Cancer after kidney transplantation in the United States. Am J Transplant 2004;4:905-13.

42 Zabierowski SE, Herlyn M. Melanoma stem cells: the dark seed of melanoma. J Clin Oncol 2008;26:2890-4.

43 Socialstyrelsen. Cancer incidence in Sweden 2010. www.socialstyrelsen.se/ publikationer2011/2011-12-15. 


\section{What is already known on this topic}

Recent reports suggest that states of immune suppression, such as after organ transplant, may be involved in the causation of malignant melanomas

Other data suggest that tumour necrosis factor (TNF) may be important for the onset and course of melanoma

Data on risk of melanoma in relation to therapeutic inhibition of TNF are limited and conflicting, as are data on whether the treated inflammatory conditions in themselves increase the risk of melanoma

\section{What this study adds}

Patients with rheumatoid arthritis treated with TNF inhibitors are at a moderately increased risk of invasive melanomas, but the overall burden of cancer in this population is not increased

A scientific need exists to further understand the role of various immunomodulatory treatments on the risk and prognosis of melanoma A clinical need exists to understand whether (or how) TNF inhibitors and other immunomodulatory drugs can be safely used in patients at high risk of melanoma

44 Askling J, Fahrbach K, Nordstrom B, Ross S, Schmid CH, Symmons D. Cancer risk with tumor necrosis factor alpha (TNF) inhibitors: meta-analysis of randomized controlled trials of adalimumab, etanercept, and infliximab using patient level data. Pharmacoepidemiol Drug Saf 2011;20:119-30.

45 Bongartz T, Sutton AJ, Sweeting MJ, Buchan I, Matteson EL, Montori V. Anti-TNF antibody therapy in rheumatoid arthritis and the risk of serious infections and malignancies: systematic review and meta-analysis of rare harmful effects in randomized controlled systematic review and meta-analsials.
trials. JAMA 2006;295:2275-85.

46 Bongartz T, Warren FC, Mines D, Matteson EL, Abrams KR, Sutton AJ. Etanercept therapy in rheumatoid arthritis and the risk of malignancies: a systematic review and individual patient data meta-analysis of randomised controlled trials. Ann Rheum Dis 2009;68:1177-83.
47 Leombruno JP, Einarson TR, Keystone EC. The safety of anti-tumour necrosis factor treatments in rheumatoid arthritis: meta and exposure-adjusted pooled analyses of serious adverse events. Ann Rheum Dis 2009;68:1136-45.

Accepted: 06 March 2013

\section{Cite this as: BMJ 2013:346:f1939}

This is an Open Access article distributed in accordance with the Creative Commons Attribution Non Commercial (CC BY-NC 3.0) license, which permits others to distribute, remix, adapt, build upon this work non-commercially, and license their derivative works on different terms, provided the original work is properly cited and the use is non-commercial. See: http://creativecommons.org/licenses/by-nc/3.0/. 


\section{Tables}

Table 1| Baseline characteristics of three population based Swedish cohorts: one cohort of rheumatoid arthritis patients starting tumour necrosis factor (TNF) inhibitor as a first ever biological drug 1998-2010, one cohort of rheumatoid arthritis patients identified 2001-10 (censored at start of first biological drug), and one matched general population comparator. Values are numbers (percentages) unless stated otherwise

Rheumatoid arthritis patients

Characteristics

TNF inhibitor treatment $(n=10$ 878) No biological drug treatment $(n=42198)$ General population $(n=162743)$

\begin{tabular}{|c|c|c|c|}
\hline Female sex & $8230(76)$ & $30232(72)$ & $116917(71)$ \\
\hline Median (IQR) age at entry (years) & $57(47-64)$ & $62(52-72)$ & $62(52-71)$ \\
\hline Median (IQR) entry year & 2005 (2002-08) & $2004(2002-07)$ & 2004 (2002-07) \\
\hline Median (IQR) follow-up (years) & $4.8(2.3-7.5)$ & $4.6(2.0-7.7)$ & $5.7(2.8-7.8)$ \\
\hline Family history of melanoma & $281(2.6)$ & $1001(2.4)$ & $4462(2.7)$ \\
\hline \multicolumn{4}{|l|}{ Country of birth: } \\
\hline Nordic & $10121(93.1)$ & $39480(93.6)$ & $150718(92.6)$ \\
\hline Other $^{*}$ & $757(6.9)$ & $2718(6.4)$ & $12025(7.4)$ \\
\hline \multicolumn{4}{|l|}{ Education,: } \\
\hline$\leq 9$ years $\dagger$ & $3211(29.5)$ & $17023(40.3)$ & $56538(34.7)$ \\
\hline$>9$ years & 7667 (70.5) & $25175(59.7)$ & $106205(65.3)$ \\
\hline \multicolumn{4}{|l|}{ Comorbidities: } \\
\hline Chronic obstructive pulmonary disease & $522(4.8)$ & $2875(6.8)$ & $5614(3.4)$ \\
\hline Non-melanoma skin cancer & $188(1.7)$ & $1034(2.5)$ & $2120(1.3)$ \\
\hline Diabetes mellitus & $893(8.2)$ & $4255(10.1)$ & $12263(7.5)$ \\
\hline Ischaemic heart disease & $1102(10.1)$ & $6932(16.4)$ & $18206(11.1)$ \\
\hline Previous joint surgery & 4314 (39.7) & $12441(29.5)$ & $12741(7.8)$ \\
\hline
\end{tabular}

IQR=interquartile range.

*Includes missing/incomplete data (approximately $1 \%$ across exposure categories).

†Includes missing/incomplete data ( $<15 \%$ across exposure categories). 
Table 2| Occurrence and hazard ratio of cancer outcomes in 42198 Swedish rheumatoid arthritis patients not treated with biological drugs, compared with matched general population cohort $(n=162743)$

\section{Events/person years}

Outcome Rheumatoid arthritis General population Hazard ratio* $(95 \% \mathrm{Cl})$

Invasive malignant melanoma† $113 / 203345$

393/854 111 $1.2(0.9$ to 1.5$)$

In situ melanoma

$57 / 197754$

$219 / 838548$

$1.2(0.9$ to 1.7$)$

Invasive all site cancer

$2788 / 196826$

9736/831 297

1.1 (1.1 to 1.2$)$

*Stratified for year of inclusion and adjusted for sex and age.

†Primary outcome. 
Table 3| Occurrence and hazard ratios of cancer outcomes in 10878 Swedish rheumatoid arthritis patients starting tumour necrosis factor inhibitor compared with 42198 not treated with biological drugs

\begin{tabular}{|c|c|c|c|c|}
\hline \multirow[b]{2}{*}{ Outcome } & \multicolumn{2}{|c|}{ Events/person year } & \multicolumn{2}{|c|}{ Hazard ratio $(95 \% \mathrm{Cl})$} \\
\hline & $\begin{array}{l}\text { Tumour necrosis factor } \\
\text { inhibitor }\end{array}$ & $\begin{array}{l}\text { No biological drug } \\
\text { treatment }\end{array}$ & $\begin{array}{l}\text { Stratified for sex and } \\
\text { adjusted for age }\end{array}$ & Fully adjusted ${ }^{*}$ \\
\hline $\begin{array}{l}\text { Invasive malignant } \\
\text { melanoma† }\end{array}$ & $38 / 57223$ & $113 / 203345$ & $1.6(1.1$ to 2.5$)$ & 1.5 (1.0 to 2.2$)$ \\
\hline In situ melanoma & $11 / 56080$ & $57 / 197754$ & $1.1(0.5$ to 2.1$)$ & - \\
\hline Invasive all site cancer & $558 / 55947$ & $2788 / 196826$ & $1.0(0.9$ to 1.1$)$ & $1.0(0.9$ to 1.1$)$ \\
\hline
\end{tabular}

*Stratified for year of inclusion and adjusted for sex, age, country of birth, personal history of non-melanoma skin cancer in situ, family history of melanoma, educational level, and comorbidities during follow-up (diabetes mellitus, ischaemic heart disease, chronic obstructive pulmonary disease, and joint surgery). †Primary outcome. 
Table 4| Number of first invasive melanomas and hazard ratios in 10878 rheumatoid arthritis patients treated with TNF inhibitor compared with 42198 not treated with biological drugs, separately by sex, age at start of TNF inhibitor, time since start of TNF inhibitor, accumulated time on TNF inhibitor treatment, and calendar period of TNF inhibitor start

\begin{tabular}{|c|c|c|}
\hline Exposure categories & Melanoma (total No of patients) & Hazard ratio* $(95 \% \mathrm{Cl})$ \\
\hline Overall & $38(10878)$ & $1.5(1.0$ to 2.2$)$ \\
\hline Women & $22(8230)$ & $1.2(0.7$ to 1.9$)(\mathrm{P}<0.001 v$ men $)$ \\
\hline Men & $16(2648)$ & $2.7(1.6$ to 4.6$)$ \\
\hline \multicolumn{3}{|c|}{ Age at start of TNF inhibitor treatment: } \\
\hline $16-49$ years & $5(3383)$ & $1.2(0.4$ to 3.2$)$ \\
\hline $50-74$ years & $31(6937)$ & $1.7(1.1$ to 2.6$)$ \\
\hline$\geq 75$ years & $2(558)$ & $1.0(0.2$ to 3.9$)$ \\
\hline \multicolumn{3}{|c|}{ Time since start of TNF inhibitor treatment: } \\
\hline$\leq 1$ year & $6(1240)$ & $1.6(0.6$ to 3.9$)$ \\
\hline$>1-5$ years & $19(4385)$ & $1.6(1.0$ to 2.8$)$ \\
\hline$>5$ years & $13(5253)$ & $1.5(0.8$ to 2.9$)$ \\
\hline \multicolumn{3}{|c|}{ Accumulated time on TNF inhibitor treatment: } \\
\hline$\leq 1$ year & $10(10878)$ & $1.5(0.8$ to 2.9$)$ \\
\hline$>1-3$ years & $14(8421)$ & $1.7(1.0$ to 3.0$)$ \\
\hline$>3-5$ years & $4(5598)$ & $0.8(0.3$ to 2.2$)$ \\
\hline$>5$ years & $10(3626)$ & $1.9(1.0$ to 3.7$)$ \\
\hline \multicolumn{3}{|c|}{ Year of first TNF inhibitor start: } \\
\hline $1998-2004$ & $25(4864)$ & $1.6(1.0$ to 2.5$)$ \\
\hline 2005-09 & $13(6014)$ & $1.5(0.8$ to 2.7$)$ \\
\hline
\end{tabular}

TNF=tumour necrosis factor.

*Stratified for year of inclusion and adjusted for sex, age, country of birth, personal history of non-melanoma skin cancer in situ, family history of melanoma, educational level, and comorbidities during follow-up (diabetes mellitus, ischaemic heart disease, chronic obstructive pulmonary disease, and joint surgery). No significant difference across exposure categories for any stratified analysis except sex (all $P>0.05$ ). 


\section{Figure}

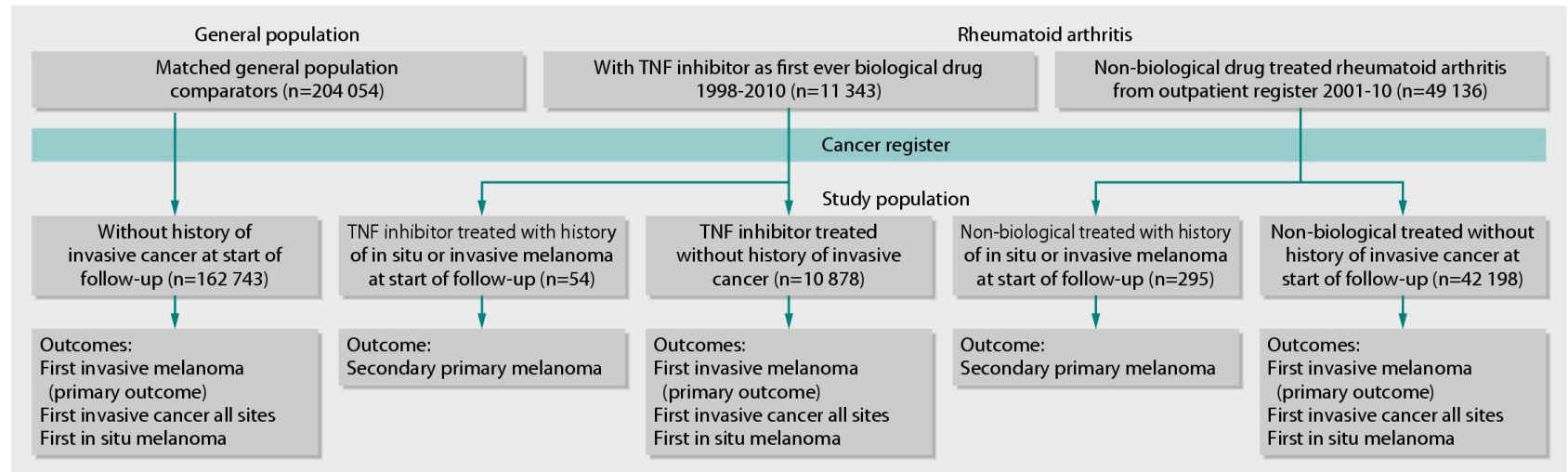

Description of study population in relation to outcome definitions. TNF=tumour necrosis factor 